\title{
Coexistence of neuronal intranuclear inclusion disease and amyotrophic lateral sclerosis: an autopsy case
}

\author{
Atsuhiko Sugiyama ${ }^{1 *} \mathbb{0}$, Takahiro Takeda ${ }^{2}$, Mizuho Koide $^{2}$, Hajime Yokota $^{3}$, Hiroki Mukai ${ }^{4}$, Yoshihisa Kitayama', \\ Kazumoto Shibuya', Nobuyuki Araki ${ }^{1}$, Ai Ishikawa², Sagiri Isose ${ }^{2}$, Kimiko Ito², Kazuhiro Honda², \\ Yoshitaka Yamanaka ${ }^{1,5}$, Terunori Sano ${ }^{6}$, Yuko Saito ${ }^{6}$, Kimihito Arai $^{2}$ and Satoshi Kuwabara ${ }^{1}$
}

\begin{abstract}
Background: Neuronal intranuclear inclusion disease (NIID) is a rare neurodegenerative disease. Pathologically, it is characterized by eosinophilic hyaline intranuclear inclusions in the cells of the visceral organs as well as central, peripheral, and autonomic nervous system cells. Recently, a GGC repeat expansion in the NOTCH2NLC gene has been identified as the etiopathological agent of NIID. Interestingly, this GGC repeat expansion was also reported in some patients with a clinical diagnosis of amyotrophic lateral sclerosis (ALS). However, there are no autopsy-confirmed cases of concurrent NIID and ALS.
\end{abstract}

Case presentation: A 60-year-old Taiwanese woman reported a four-month history of progressive weakness beginning in the right foot that spread to all four extremities. She was diagnosed with ALS because she met the revised El Escorial diagnostic criteria for definite ALS with upper and lower motor neuron involvement in the cervical, thoracic, and lumbosacral regions. She died of respiratory failure at 22 months from ALS onset, at the age of 62 years. Brain magnetic resonance imaging (MRI) revealed lesions in the medial part of the cerebellar hemisphere, right beside the vermis (paravermal lesions). The subclinical neuropathy, indicated by a nerve conduction study (NCS), prompted a potential diagnosis of NIID. Antemortem skin biopsy and autopsy confirmed the coexistence of pathology consistent with both ALS and NIID. We observed neither eccentric distribution of p62-positive intranuclear inclusions in the areas with abundant large motor neurons nor cytopathological coexistence of ALS and NIID pathology in motor neurons. This finding suggested that ALS and NIID developed independently in this patient.

Conclusions: We describe a case of concurrent NIID and ALS discovered during an autopsy. Abnormal brain MRI findings, including paravermal lesions, could indicate the coexistence of NIID even in patients with ALS showing characteristic clinical phenotypes.

Keywords: Neuronal intranuclear inclusion disease, Amyotrophic lateral sclerosis, Magnetic resonance imaging, Autopsy, Paravermal lesion, Case report

${ }^{*}$ Correspondence: asugiyama@chiba-u.jp

${ }^{1}$ Department of Neurology, Graduate School of Medicine, Chiba

University, 1-8-1 Inohana, Chuo-ku, Chiba 260-8677, Japan

Full list of author information is available at the end of the article

\section{Background}

Neuronal intranuclear inclusion disease (NIID), a rare neurodegenerative disease, is pathologically characterized by eosinophilic hyaline intranuclear inclusions in the cells of the visceral organs as well as central, peripheral, and autonomic nervous system cells [1,2]. NIID's clinical 
manifestations vary and include cerebellar ataxia, pyramidal and extrapyramidal symptoms, peripheral neuropathy, autonomic dysfunction, cognitive dysfunction, and retinopathy $[1,2]$. It appears in both familial and sporadic forms, and onset can occur at any age $[1,2]$.

Recently, techniques such as long-read sequencing [3, 4] and direct identification by short-read analysis [5] have confirmed a GGC repeat expansion in the NOTCH2NLC gene as NIID's underlying etiopathology. This abnormal GGC repeat expansion in the NOTCH2NLC gene is reportedly associated with essential tremor and leukoencephalopathy $[6,7]$. Moreover, this GGC repeat expansion in the NOTCH2NLC gene has appeared in 4 out of 545 patients with a clinical diagnosis of amyotrophic lateral sclerosis (ALS) [8]. These observations suggest the following hypotheses: (i) ALS might be a special NIID phenotype, and the GGC repeat expansion might modify the clinical manifestations of ALS, or (ii) NIID and ALS might share part(s) of the pathophysiology and can, therefore, coexist. Interestingly, no autopsy-confirmed cases of concurrent NIID and ALS were previously reported.

Here, we describe a case wherein the main clinical features were consistent with ALS diagnosis, but an autopsy confirmed the coexistence of both NIID and ALS.

\section{Case presentation}

A 60-year-old Taiwanese woman presented to the Chiba University Hospital with a four-month history of progressive weakness beginning in the right foot and spreading to all four extremities. Her medical history was unremarkable except for cough-variant asthma, and there was no family history of neuromuscular or neurodegenerative disorders.

A neurological examination showed no abnormality of the cranial nerves except for slight dysarthria. However, reduced muscle strength in all four extremities, especially in the right foot, with wasting, was observed. Fasciculations were noted in both arms. Deep tendon reflexes were brisk with a positive Trömner sign on the right, and bilateral plantar reflexes were extensor. Abdominal skin reflexes were absent bilaterally, whereas abdominal muscle reflex was enhanced. There were no signs of cerebellar dysfunction. The sensation was also intact to all modalities used for testing. The patient scored 28/30 on the Mini-Mental State Examination (MMSE) as she lost points for recall and following a command. She scored 16/18 on the Frontal Assessment Battery (FAB) where she lost points for similarities and lexical fluency. The overall score of Addenbrooke's Cognitive Examination III was 83 out of 100 , with subscores of $18 / 18$ for attention and orientation, $15 / 26$ for memory, $9 / 14$ for fluency, $25 / 26$ for language, and $16 / 16$ for visuospatial skills.

Needle electromyography demonstrated active and chronic denervation potentials in her biceps brachii, first dorsal interosseous, thoracic paraspinal muscles, tibialis anterior, and vastus lateralis. Nerve conduction studies (NCS) in the right upper and lower limbs revealed reduced conduction velocities and prolonged distal latencies in all tested motor nerves (Table 1). Decreased compound muscle action potential was observed in the ulnar, the peroneal, and the tibial nerves. Sensory studies revealed reduced conduction velocities and decreased sensory nerve action potential in the ulnar nerve. Brain magnetic resonance imaging (MRI) revealed bilateral subcortical high-intensity lesions in the precentral gyri and abnormal high-intensity signals along the corticospinal tracts on T2-weighted (T2WI) and fluid-attenuated inversion recovery (FLAIR) images (Fig. 1). High-intensity signals in the medial part of the cerebellar hemisphere, right beside the vermis (paravermal lesions), were also seen in FLAIR images (Fig. 1).

The patient met the El Escorial diagnostic criteria for definite ALS with upper and lower motor neuron

Table 1 Results of nerve conduction studies in the right upper and lower limbs at the age of 60 years

\begin{tabular}{|c|c|c|c|c|c|c|}
\hline & \multicolumn{4}{|l|}{ Motor nerve } & \multicolumn{2}{|l|}{ Sensory nerve } \\
\hline & Distal latency (ms) & $\begin{array}{l}\text { Conduction } \\
\text { velocity }(\mathrm{m} / \mathrm{s})\end{array}$ & CMAP $(\mathrm{mV})$ & F-wave latency (ms) & $\begin{array}{l}\text { Conduction } \\
\text { velocity }(\mathrm{m} / \mathrm{s})\end{array}$ & SNAP $(\mu \mathrm{V})$ \\
\hline R. Median nerve & $\underline{4.7(<4.5)}$ & $\underline{43(>48)}$ & $5.55(>5.0)$ & $\underline{35.9(<31.4)}$ & $\underline{42(>43)}$ & $\underline{11(>11.3)}$ \\
\hline R. Ulnar nerve & $\underline{5.0(<3.6)}$ & $\underline{40(>46)}$ & $\underline{3.42(>4.7)}$ & $\underline{41.8(<31.7)}$ & $43(>40)$ & $27(>8.8)$ \\
\hline R. Peroneal nerve & $\underline{6.2(<6.2)}$ & $35(>37.1)$ & $\underline{0.58(>0.7)}$ & $\underline{65.1(<55.3)}$ & & \\
\hline R. Tibial nerve & $\underline{6.1(<5.9)}$ & $\underline{31(>36)}$ & $\underline{3.30(>5.6)}$ & $\underline{60.1(<56.8)}$ & & \\
\hline R. Sural nerve & & & & & $40(>37)$ & $12(>3.4)$ \\
\hline
\end{tabular}

All studies were performed in the right extremities (R.)

All sensory studies were antidromically measured

Abnormal values are indicated with underlines. Normal limits are indicated in parentheses (our laboratory data)

CMAP compound muscle action potential, SNAP sensory nerve action potential 


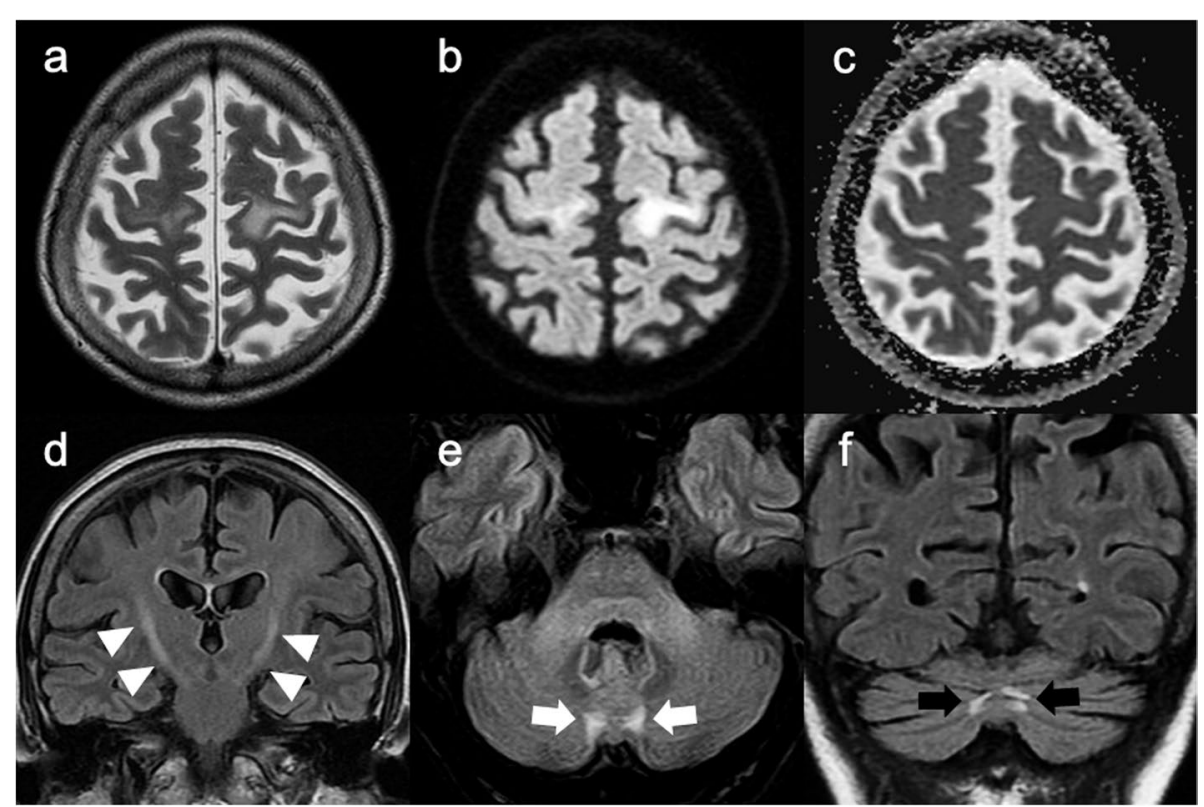

Fig. 1 Brain magnetic resonance imaging (MRI) at the age of 60. Brain MRI shows bilateral subcortical high-intensity signals in the precentral gyri on T2-weighted (a) and diffusion-weighted (b) images. These subcortical legions were isointense on apparent coefficient mapping (c). Abnormal high-intensity signals along the corticospinal tracts are observed bilaterally on FLAIR image (d, white arrowheads). High signal intensities in the medial part of the cerebellar hemisphere right beside the vermis (paravermal lesions) are also seen bilaterally on axial (e, white arrows) and coronal (f, black arrows) FLAIR images

involvement in the cervical, thoracic, and lumbosacral regions [9]. On the other hand, paravermal lesions on brain MRI and NCS findings that indicated demyelinating and axonal neuropathy prompted a diagnosis of NIID. A skin biopsy revealed eosinophilic, p62-positive, ubiquitin-positive, intranuclear inclusions in adipocytes, sweat gland cells, fibroblasts, and Schwann cells of the endoneurium and the perineurium. Electron microscopy showed that they consisted of tubule-filamentous material (Fig. 2). Based on these results, we considered the following possible diagnoses, namely, (i) coexistence of ALS and NIID or (ii) an ALS phenotype of NIID; however, a definitive diagnosis was difficult to establish at that time.

Subsequently, clinical signs of upper and lower motor neuron involvement in the bulbar lesion became evident. The patient underwent a gastrostomy eight months after ALS onset and died 22 months after onset, at 62 years of age, due to respiratory failure.

The patient's family provided informed consent for postmortem analyses, and an autopsy was performed. Appropriate brain areas were dissected and embedded in paraffin, $5 \mu \mathrm{m}$-thick serial sections were stained with hematoxylin and eosin and Klüver-Barrera (KB), and then immunostained against phosphorylated transactivation response DNA-binding protein (TARDBP) $43 \mathrm{kDa}$ (pTDP-43) (pS409/410-2, polyclonal, rabbit, Cosmo Bio, Tokyo, Japan, 1:3,000), TDP-43 (clone 3H8, monoclonal, mouse, Novus Biologicals, Centennial, CO, 1:3,000) and p62 (SQSTM1, polyclonal, rabbit, MBL, Nagoya, Japan).

At autopsy, the brain weighed 1,020 g. The pia mater, particularly on the central regions' surface, appeared cloudy, suggesting cortical atrophy in these areas (Fig. 3). The precentral cortex appeared more brown than usual and displayed an ill-defined corticomedullary boundary, which was in contrast to the other cortices (Fig. 3). The spinal cord was atrophic with clear evidence of thinning in its anterior roots. Microscopic examination was remarkable for gliosis and loss of spinal motor neurons. Bunina bodies and TDP-43-positive cytoplasmic inclusions were occasionally identified in the remaining motor neurons of the anterior spinal horn and in the hypoglossal nucleus (Fig. 3). TDP-43-positive cytoplasmic inclusions were absent from the neocortex, limbic areas, and subcortical gray matter, including in the striatum, pallidum, and thalamus. Precentral gyrus thinning was corroborated by severe neuronal loss and gliosis, and the precentral subcortical white matter showed severe gliosis and the presence of several vacuoles (Fig. 3). Vacuolar degeneration, accompanied by macrophage infiltration, occurred throughout the corticospinal tracts, such as in the posterior limb of the internal capsule, the cerebral peduncle, the pyramid of the medulla oblongata, and the lateral funiculus of the spinal cord (Fig. 3). We also observed that significant quantities of p62-positive 

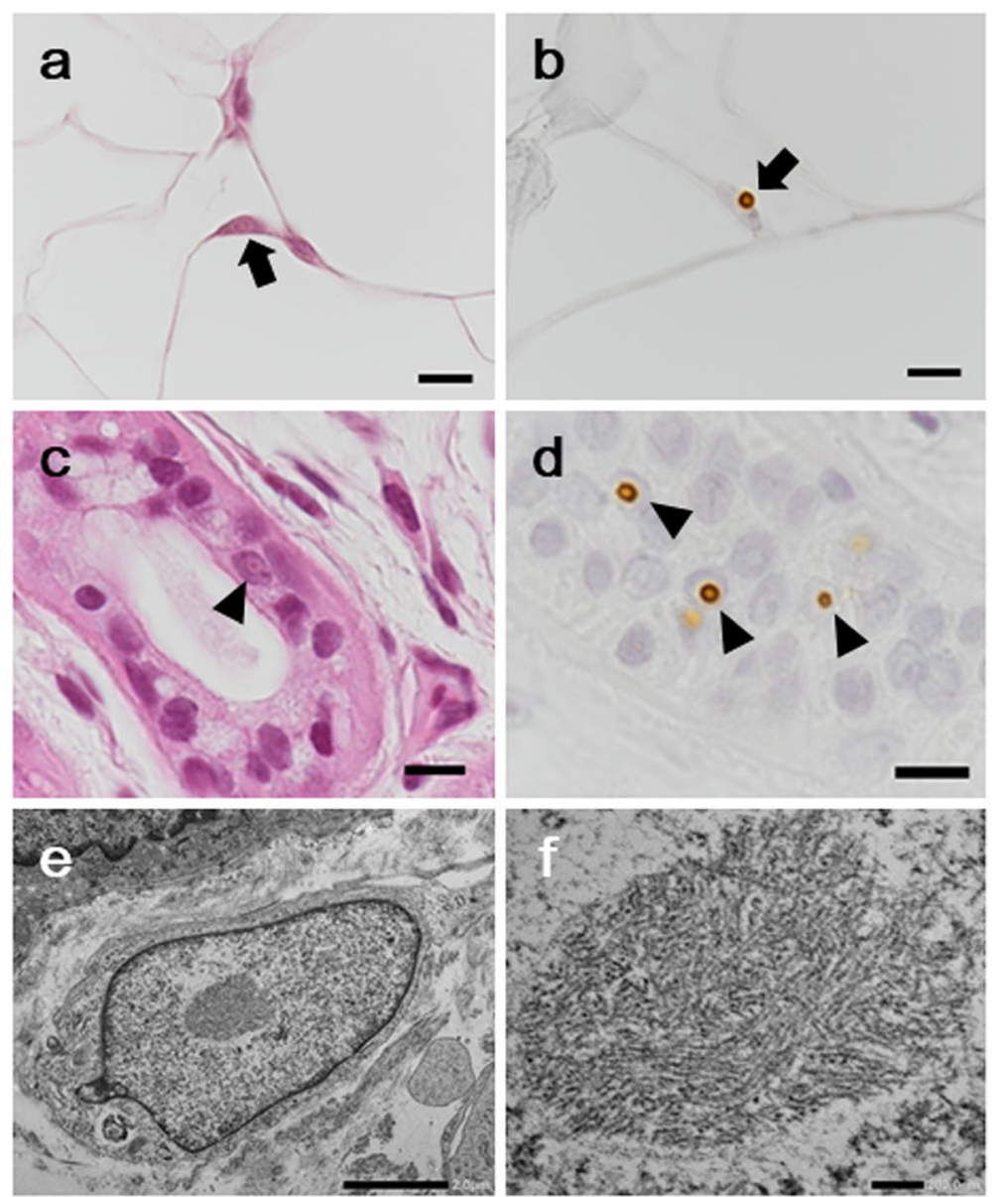

Fig. 2 Skin biopsy findings at the age of 60. Eosinophilic and p62-positive intranuclear inclusions in adipocytes (a, b) and sweat gland cells (c, d). Electron microscopy of an inclusion body in the fibroblast nucleus revealed that it consisted of tubule-filamentous material but without a limiting membrane (e, f). Staining: a, c hematoxylin and eosin; b, d anti-p62 immunohistochemical staining. Scale bars: a-d $10 \mu \mathrm{m} ; \mathbf{e} 2 \mu \mathrm{m} ; \mathbf{f} 200 \mathrm{~nm}$

intranuclear inclusions were widely distributed across different central nervous system areas, including the neocortex, the limbic regions, basal ganglia, brainstem, cerebellum, and the spinal cord (Fig. 4). Most inclusions appeared to be present in the glial cells (astrocytes, oligodendrocytes, and ependymal cells), were well-defined and round, and appeared eosinophilic with hyaline-like features on hematoxylin-eosin staining. A small number of p62-positive inclusions were localized to the nuclei of motor neurons, and they were plotted on a brain map at $\times 200$ magnification using a VS120 virtual slide system (Olympus, Tokyo, Japan) to investigate an association, if any, between the distribution of p62-positive intranuclear inclusions and neuronal degeneration (Fig. 5). We found no eccentric distribution of these inclusions in areas with abundant large motor neurons, such as in the spinal cord's anterior horn and the deep Betz cell layer of the precentral gyrus. Additionally, double immunohistochemical evaluation using antibodies against p62 and TDP-43 on five different sections at the fourth level of the lumbar cord to determine the cytopathological coexistence of motoneuronal p62-positive intranuclear inclusions (NIID pathology) and pTDP-43-positive cytoplasmic inclusions with native TDP-43 loss from the nucleus (ALS pathology) did not show any motor neuron overlap between NIID and ALS pathology (Fig. 6). These results indicated that, in the present case, ALS pathology might have occurred independently of NIID pathology.

We observed abundant p62-positive intranuclear inclusions in the cerebellar glial cells, particularly in the Purkinje and the granular cell layers and in the periventricular ependymal region (Fig. 4). Inclusions were also occasionally identified in cerebellar cortical neurons, such as in the Purkinje and granular cells; however, neurons harboring p62-positive intranuclear inclusions in the dentate nucleus were rare. The distribution of intranuclear inclusions in the glia varied across different areas of white matter. These inclusions were 

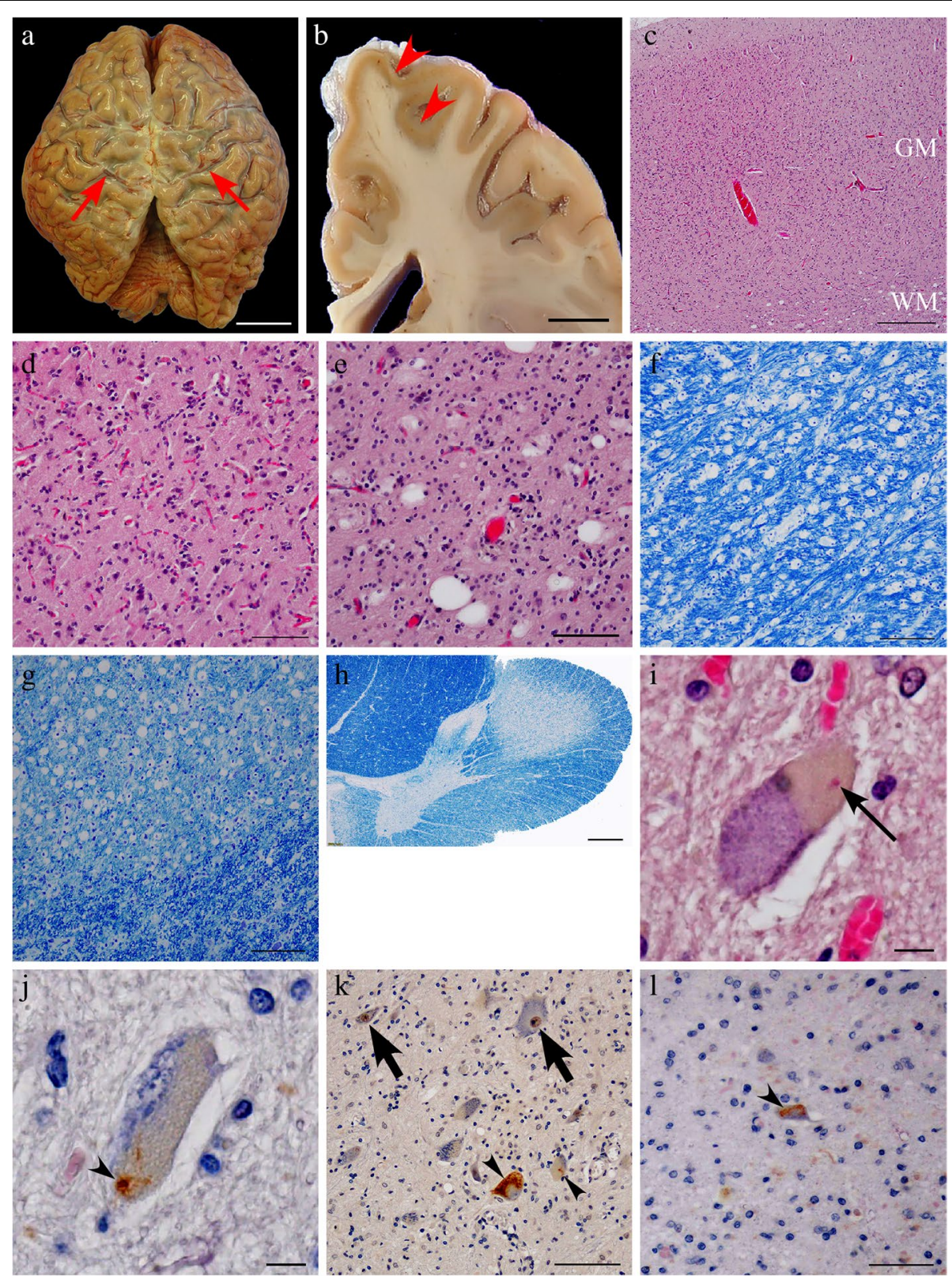

Fig. 3 Autopsy findings of amyotrophic lateral sclerosis (ALS) pathology. Top view of the brain before fixation. Cloudiness of the pia mater was notable, especially on the surface of the central region, suggesting cortical atrophy around the central sulci (red arrows) (a). The primary motor cortex (red arrowheads) exhibits a dark-brownish color, with the border's fuzziness at the corticomedullary junction (b). The primary motor cortex's low-power view shows cortical thinning and vacuolar formation in the white matter just beneath the cortex (c). Cortical degeneration associated with severe neuronal loss and gliosis (d). There were many vacuoles in the subcortical white matter of the primary motor cortex. The vacuoles appear to be isolated from each other (e). Klüver-Barrera (KB) staining at the level of the internal capsule (f) and the pyramid of the medulla oblongata ( $\mathbf{g}$ ) demonstrate microvacuolar formation with infiltration of macrophages. KB staining in the thoracic spinal cord revealed corticospinal tract degeneration (h). Bunina body (thin black arrow) (i) and transactivation reaction DNA-binding protein 43 kDa (TDP-43)-positive cytoplasmic inclusions (black arrowheads) in the remaining motor neurons in the anterior spinal horn (j, $\mathbf{k})$ and in the hypoglossal nuclei (I) are shown. Native TDP-43 was preserved in the nuclei of normal motor neurons (k, black arrows). GM: gray matter, WM: white matter. Scale bars $=\mathbf{a} 3 \mathrm{~cm}, \mathbf{b} 1 \mathrm{~cm}, \mathbf{c}, \mathbf{h}$ $500 \mu \mathrm{m}, \mathbf{d}-\mathbf{g} 100 \mu \mathrm{m}, \mathbf{i}, \mathbf{j} 10 \mu \mathrm{m}$ 

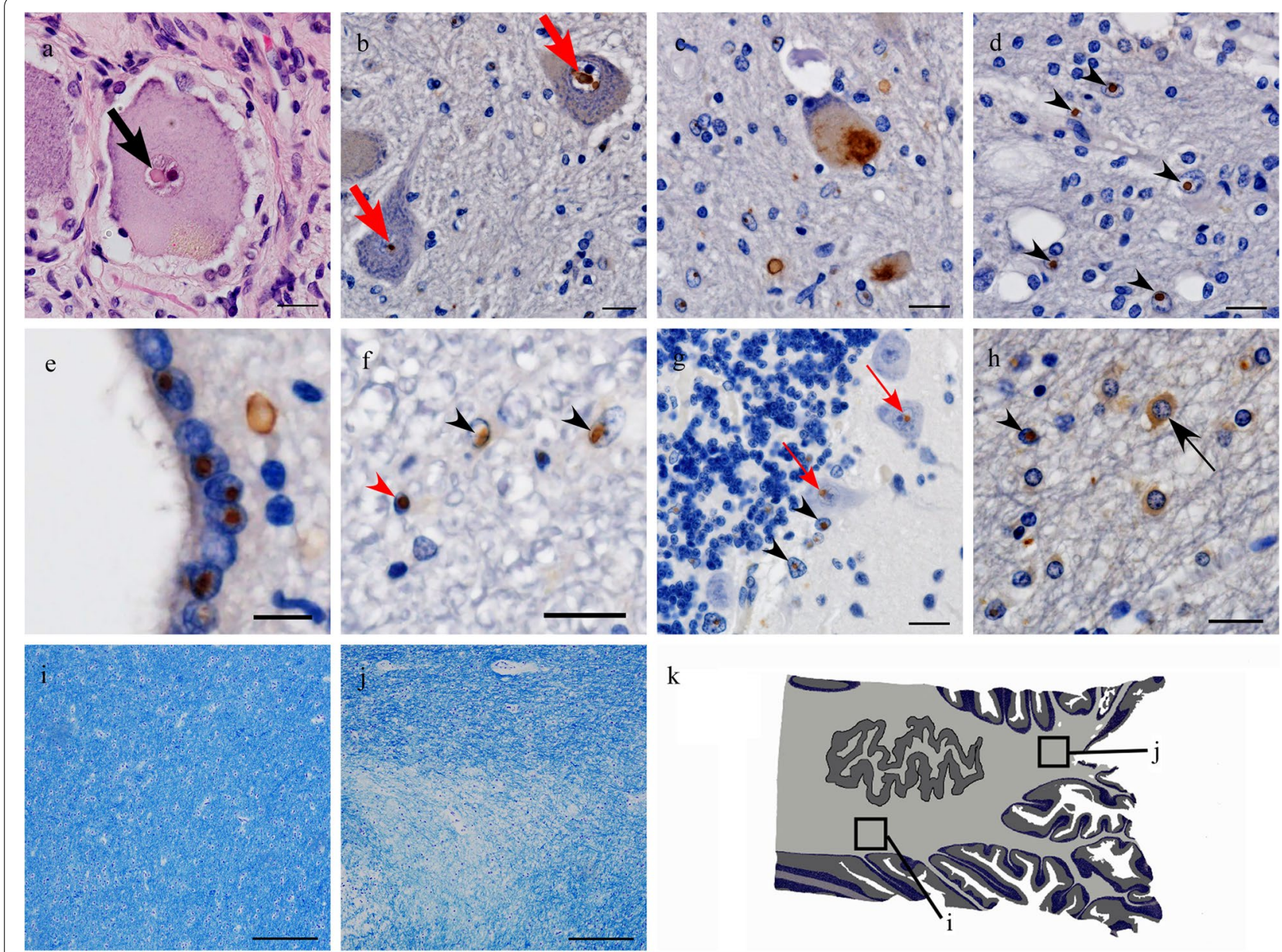

$\mathrm{k}$

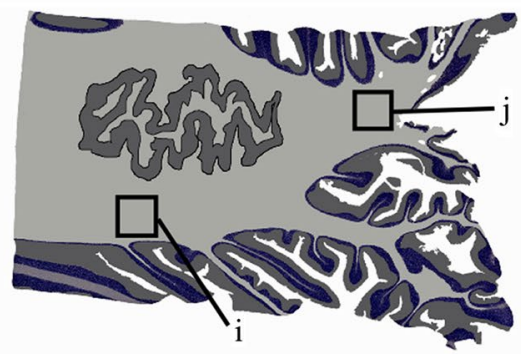

Fig. 4 Autopsy findings of neuronal intranuclear inclusion disease (NIID) pathology. Small hyaline-like inclusions were observed in the nucleus of the posterior ganglion cell (black arrow) (a). The motor neurons harboring intranuclear (red arrows) (b) and intracytoplasmic (c) inclusions that were immunopositive for p62 are shown. p62-positive intranuclear inclusions in astrocytes, around vacuole formation in the white matter of precentral gyrus (black arrowheads) (d). Periventricular ependymal cells have high prevalence of p62-positive intranuclear inclusions (e). Intranuclear inclusions immunopositive for p62 were observed in the astrocytes (black arrowheads) and oligodendrocytes (red arrowhead) in the spinal cord (f). The photograph (g) shows p62-positive intranuclear inclusions in glial cells of the cerebellar cortex (black arrowhead) and Purkinje cells (thin red arrows). In addition to the intranuclear inclusions (black arrowhead), intracytoplasmic glial inclusions immunopositive for p62 (thin black arrow) were occasionally found in the severely affected white matter lesions in the cerebellum (h). Normal (i) and demyelinated (j) white matter of the cerebellum are shown in KB stains, square areas in $(\mathbf{k})$, which are a schematic representation at the level of the dentate nucleus. Scale bars $=\mathbf{a}-\mathbf{d}, \mathbf{f}-\mathbf{h}$ $20 \mu \mathrm{m}, \mathbf{e} 10 \mu \mathrm{m}, \mathbf{i}, \mathbf{j} 200 \mu \mathrm{m}$

more abundant in the paravermis' white matter than in the hemispheric white matter (Fig. 5). Interestingly, the p62-positive nuclear inclusions in the paravermal white matter were accompanied by focal demyelination (Fig. 4). This lesion appeared to correspond with paravermal high-intensity lesions observed on MR images (Fig. 1).

\section{Discussion and conclusions}

The patient presented with a clinical course consistent with limb-onset ALS and met the El Escorial diagnostic criteria for a definite antemortem ALS diagnosis. Paravermal lesions in the medial part of the cerebellar hemisphere as seen in the brain MRI prompted a diagnosis of NIID, and both antemortem skin biopsy and postmortem autopsy confirmed the coexistence of ALS and NIID. Further, the absence of an eccentric distribution of p62-positive intranuclear inclusions in areas with abundant large motor neurons and no evidence of cytopathological coexistence of ALS and NIID pathology in the motor neurons suggests that ALS and NIID developed independently in this patient.

ALS and NIID may share underlying etiologic factors but coexist independently in an individual. Intranuclear inclusions positive for ubiquitin and p62 have been reported in both ALS and NIID [1, 2, 10]. Further, 
a

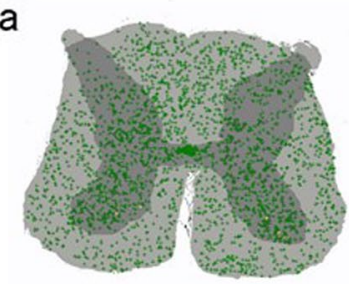

C

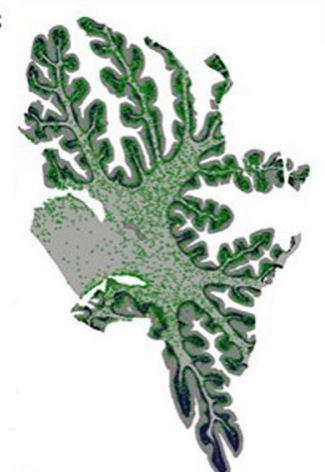

b

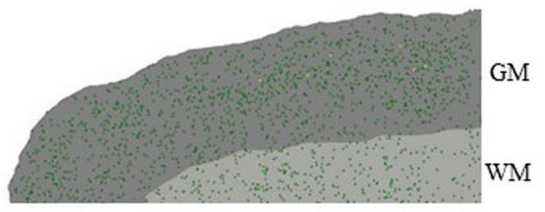

d

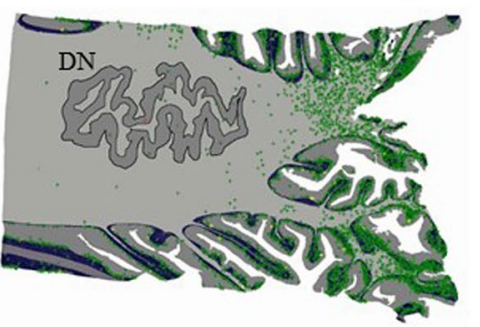

e

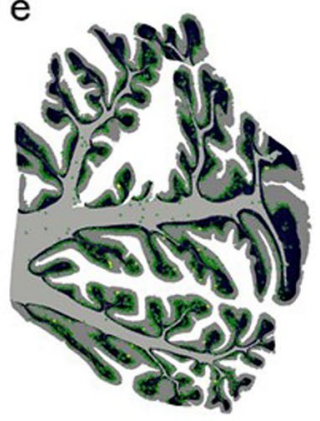

Fig. 5 Topographical distribution of p62-positive intranuclear inclusions. Plotting scheme representing a topographical distribution of p62-positive intranuclear inclusions on sections at the level of the 4th lumbar cord (a), the primary motor cortex (b), and cerebellum at the level of the paravermis (c), dentate nucleus (d), and hemisphere (e). There is no eccentric distribution of p62-positive intranuclear inclusions to the areas with abundant large motor neurons, including the anterior horn of the spinal cord (a) and the deeper zone of the primary motor cortex (b). In the cerebellum, in contrast to the motor-related area with lack of eccentric distribution, the p62-positive intranuclear inclusions were predominantly distributed in the medial (paravermal) white matter (c)(d) rather than the lateral (hemispheric) white matter (e). DN: dentate nucleus, GM: gray matter, WM: white matter

GGC repeat expansion in the NOTCH2NLC gene, which is etiopathological of NIID, has been observed in 4 out of 545 patients with a clinical diagnosis of ALS [8]. While the previous report did not describe the definitive pathological diagnoses in these four patients, they reported eosinophilic, p62-positive, ubiquitin-positive intranuclear inclusions in skin biopsies in two of them [8]. Unfortunately, we could not perform genetic analysis of the NOTCH2NLC gene for this case; nevertheless, the autopsy confirmed the coexistence of pathology consistent with both ALS and NIID. Moreover, the distribution of p62-positive intranuclear inclusions and double immunohistochemical staining against p62 and TDP-43 suggested that ALS and NIID had developed independently in our patient. While ALS and NIID's coincidental coexistence cannot be completely ruled out, the very low prevalence of these two conditions precludes any assessment of the probability of such an occurrence.

It is possible that paravermal lesions in the medial part of the cerebellar hemisphere are the sole radiological clue for diagnosing NIID because, in our case, although the brain MRI revealed abnormal signals in the paravermal areas, findings typical of NIID, such as high-intensity signals along the corticomedullary junction on diffusionweighted imaging (DWI) or diffuse high-intensity signals in cerebral white matter on FLAIR [2, 11], were absent. The autopsy also revealed focal demyelination with abundant p62-positive nuclear inclusions in the white matter of the paravermis, which appeared to correspond to the paravermal lesions in the MRI. Further, paravermal lesions have been described as features of adult-onset NIID [11]. Interestingly, observations similar to our case, i.e., paravermal lesions without apparent DWI abnormality along the corticomedullary junction on MRI, at the time of onset, have been previously described [12]. In addition to these paravermal lesions, MRI also revealed abnormal signals in the precentral subcortical white matter and along the corticospinal tract. These lesions appeared to correspond to vacuolar degeneration seen during autopsy pathology. Consistent with our observations, previous studies associated vacuolar degeneration with abnormal MRI signals in the precentral subcortical white matter and along the corticospinal tract [13].

The results of NCS in our case may be interpreted as a mixture of NIID-associated subclinical peripheral neuropathy and ALS-related changes. Motor and peripheral sensory neuropathy is a common symptom in patients with NIID [2] and can be the predominant symptom in some NIID patients [14]. Slower conduction in both motor and sensory nerves has been 
Non-phospholyrated TDP-43
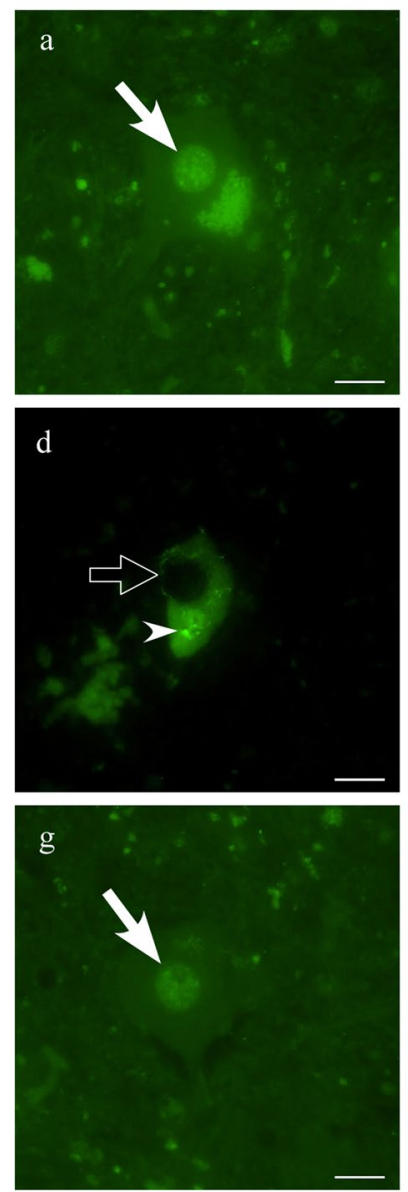

p62
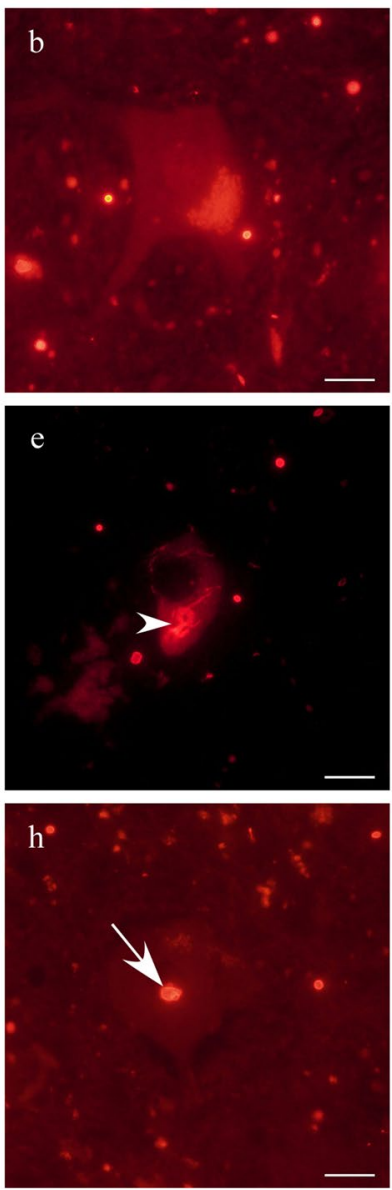

Merge
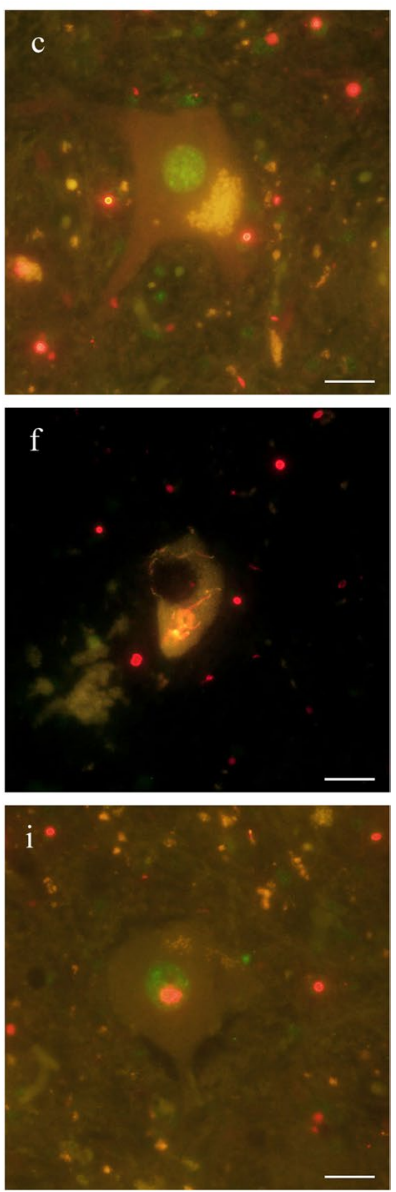

Fig. 6 Double immunohistochemical evaluation using antibodies against TDP-43 and p62. To evaluate whether each cytopathology (ALS and NIID pathology) may coexist in a motor neuron, a double immunofluorescence study was performed using antibodies against non-phosphorylated TDP-43 and p62. The photographs (a-c) show a normal motor neuron with preservation of native TDP-43 in the nucleus (arrow). The photographs (d-f) demonstrate a motor neuron with skein-like inclusion (arrowhead), both positive for TDP-43 and p62, along with loss of nuclear native TDP-43 (punched-out arrow). The photographs (g-i) showing p62-positive inclusion (thin arrow) in the nucleus of a motor neuron with remaining native TDP-43 (arrow) in the nucleus. $\mathbf{a}, \mathbf{d}, \mathbf{g}$ TDP-43, b, e, $\mathbf{h}$ p62 and $\mathbf{c}, \mathbf{f}, \mathbf{i}$ merge. Scale bars $=20 \mu \mathrm{m}$

reported to be frequently observed in patients with NIID [2], and prolonged distal latencies in motor nerves, along with slower conduction in sensory nerves, as seen in our case, might be associated with NIID. The revised El Escorial criteria for ALS diagnosis allow abnormal sensory NCS only in the presence of entrapment syndrome or coexisting peripheral nerve disease. While normal sensory NCS has been shown in ALS patients $[9,15]$, motor or sensory nerve conduction abnormalities do occur in some ALS patients [16-18].

This case report's limitations are that the GGC repeat length of the NOTCH2NLC gene and CGG repeat length of the FMR1 gene could not be determined.
Similarities in clinical, radiological, and pathological findings between NIID and fragile X- associated tremor/ataxia syndrome (FXTAS) have been reported $[19,20]$, and genetic testing is recommended to discriminate between these entities [2, 21]. However, pathological differences between NIID and FXTAS have also been reported [21], and the non-ALS pathologic findings were attributed to NIID rather than FXTAS, based on the following points. First, intranuclear inclusions were observed in oligodendrocytes, but such inclusions are not observed in FXTAS [22]. Second, there were intranuclear inclusions in adipocytes, sweat gland cells, and fibroblasts. The usefulness of skin biopsies has been reported in NIID but not in FXTAS [23, 24]. 
In conclusion, we describe a case of coexisting NIID and ALS diagnosed at autopsy. Abnormal brain MRI findings, including paravermal lesions, could indicate the coexistence of NIID even in patients with ALS showing a typical clinical phenotype.

\section{Abbreviations}

NIID: Neuronal intranuclear inclusion disease; ALS: Amyotrophic lateral sclerosis; MMSE: Mini-Mental State Examination; FAB: Frontal Assessment Battery; NCS: Nerve conduction study; MRI: Magnetic resonance imaging; FLAIR: Fluidattenuated inversion recovery; TDP-43: Transactivation response DNA-binding protein 43 kDa; DWI: Diffusion-weighted imaging; FXTAS: Fragile X-associated tremor/ataxia syndrome.

\section{Acknowledgements}

We would like to thank the patient for her participation in this study. We are grateful to the pathological team and Mrs. Yuriko Ogawa at National Hospital Organization Chibahigashi National Hospital for their excellent technical assistance.

\section{Authors' contributions}

AS: Study concept and writing the manuscript; TT: Writing the manuscript and performing histological examination; MK: Obtaining the clinical information, editing the manuscript, and conception of the study; HY: Acquisition and evaluating the MRI data and critical revision of the manuscript for important intellectual content, HM: Acquisition and evaluating the MRI data and critical revision of the manuscript for important intellectual content; YK: Obtaining the clinical information, editing the manuscript, and conception of the study; KS: Critical revision of the manuscript for important intellectual content; NA: Critical revision of the manuscript for important intellectual content; Al: Critical revision of the manuscript for important intellectual content; SI: Critical revision of the manuscript for important intellectual content; Kl: Critical revision of the manuscript for important intellectual content; $\mathrm{KH}$ : Critical revision of the manuscript for important intellectual content; YY: Critical revision of the manuscript for important intellectual content; TS: Performing histological examination, editing the manuscript, and conception of the study; YS: Performing histological examination and editing the manuscript; $\mathrm{KA}$ : Critical revision of the manuscript for important intellectual content; SK: Conception and design of the study, and revision of the manuscript. All authors read and approved the final manuscript.

\section{Funding}

Not applicable.

\section{Availability of data and materials}

The complete data are available from the corresponding author on reasonable request.

\section{Declarations}

\section{Ethics approval and consent to participate}

Not applicable.

\section{Consent for publication}

The written informed consent has been obtained from the patient's family for publication of this case report and any accompanying images.

\section{Competing interests}

The authors declare no conflicts of interest associated with this manuscript.

\section{Author details}

${ }^{1}$ Department of Neurology, Graduate School of Medicine, Chiba University, 1-8-1 Inohana, Chuo-ku, Chiba 260-8677, Japan. ${ }^{2}$ Department of Neurology, National Hospital Organization Chibahigashi National Hospital, Chiba, Japan. ${ }^{3}$ Department of Diagnostic Radiology and Radiation Oncology, Graduate School of Medicine, Chiba University, Chiba, Japan. ${ }^{4}$ Department of Radiology, Chiba University Hospital, Chiba, Japan. ${ }^{5}$ Urayasu Rehabilitation Education
Center, Chiba University Hospital, Chiba, Japan. ${ }^{6}$ Department of Pathology and Laboratory Medicine, National Center of Neurology and Psychiatry, Tokyo, Japan.

Received: 8 March 2021 Accepted: 29 June 2021

Published online: 09 July 2021

\section{References}

1. Takahashi-Fujigasaki J. Neuronal intranuclear hyaline inclusion disease. Neuropathology. 2003;23:351-9

2. Sone J, Mori K, Inagaki T, Katsumata R, Takagi S, Yokoi S, Araki K, Kato T, Nakamura T, Koike H, Takashima H, Hashiguchi A, Kohno Y, Kurashige T, Kuriyama M, Takiyama Y, Tsuchiya M, Kitagawa N, Kawamoto M, Yoshimura H, Suto Y, Nakayasu H, Uehara N, Sugiyama H, Takahashi M, Kokubun N, Konno T, Katsuno M, Tanaka F, Iwasaki Y, Yoshida M, Sobue G. Clinicopathological features of adult-onset neuronal intranuclear inclusion disease. Brain. 2016;139:3170-86.

3. Sone J, Mitsuhashi S, Fujita A, Mizuguchi T, Hamanaka K, Mori K, Koike H, Hashiguchi A, Takashima H, Sugiyama H, Kohno Y, Takiyama Y, Maeda K, Doi H, Koyano S, Takeuchi H, Kawamoto M, Kohara N, Ando T, leda T, Kita Y, Kokubun N, Tsuboi Y, Katoh K, Kino Y, Katsuno M, Iwasaki Y, Yoshida M, Tanaka F, Suzuki IK, Frith MC, Matsumoto N, Sobue G. Long-read sequencing identifies GGC repeat expansions in NOTCH2NLC associated with neuronal intranuclear inclusion disease. Nat Genet. 2019;51:1215-21.

4. Tian Y, Wang JL, Huang W, Zeng S, Jiao B, Liu Z, Chen Z, Li Y, Wang Y, Min HX, Wang XJ, You Y, Zhang RX, Chen XY, Yi F, Zhou YF, Long HY, Zhou CJ, Hou X, Wang JP, Xie B, Liang F, Yang ZY, Sun QY, Allen EG, Shafik AM, Kong $H E$, Guo JF, Yan XX, Hu ZM, Xia K, Jiang H, Xu HW, Duan RH, Jin P, Tang BS, Shen $L$. Expansion of human-specific GGC repeat in neuronal intranuclear inclusion disease-related disorders. Am J Hum Genet. 2019;105:166-76.

5. Takahashi Y, Ichikawa Y, Mano T, Iwata A, Harigaya Y, Matsukawa MK, Matsukawa T, Tanaka M, Shirota Y, Ohtomo R, Kowa H, Date H, Mitsue A, Hatsuta H, Morimoto S, Murayama S, Shiio Y, Saito Y, Mitsutake A, Kawai M, Sasaki T, Sugiyama Y, Hamada M, Ohtomo G, Terao Y, Nakazato Y, Takeda A, Sakiyama Y, Umeda-Kameyama Y, Shinmi J, Ogata K, Kohno Y, Lim SY, Tan AH, Shimizu J, Goto J, Nishino I, Toda T, Morishita S, Tsuji S. Noncoding CGG repeat expansions in neuronal intranuclear inclusion disease, oculopharyngodistal myopathy and an overlapping disease. Nat Genet. 2019;51:1222-32.

6. Sun QY, Xu Q, Tian Y, Hu ZM, Qin LX, Yang JX, Huang W, Xue J, Li JC, Zeng S, Wang Y, Min HX, Chen XY, Wang JP, Xie B, Liang F, Zhang HN, Wang CY, Lei LF, Yan XX, Xu HW, Duan RH, Xia K, Liu JY, Jiang H, Shen L, Guo JF, Tang BS. Expansion of GGC repeat in the human-specific NOTCH2NLC gene is associated with essential tremor. Brain. 2020;143:222-33.

7. Okubo M, Doi H, Fukai R, Fujita A, Mitsuhashi S, Hashiguchi S, Kishida H, Ueda N, Morihara K, Ogasawara A, Kawamoto Y, Takahashi T, Takahashi K, Nakamura H, Kunii M, Tada M, Katsumoto A, Fukuda H, Mizuguchi T, Miyatake S, Miyake N, Suzuki J, Ito Y, Sone J, Sobue G, Takeuchi H, Matsumoto N, Tanaka F. GGC repeat expansion of NOTCH2NLC in adult patients with leukoencephalopathy. Ann Neurol. 2019;86:962-8.

8. Yuan Y, Liu Z, Hou X, Li W, Ni J, Huang L, Hu Y, Liu P, Hou X, Xue J, Sun O Tian $Y$, Jiao B, Duan $R$, Jiang $H$, Shen L, Tang B, Wang J. Identification of GGC repeat expansion in the NOTCH2NLC gene in amyotrophic lateral sclerosis. Neurology. 2020;95:e3394-405.

9. Brooks BR, Miller RG, Swash M, Munsat TL, World Federation of Neurology Research Group on Motor Neuron Diseases. El Escorial revisted: revised criteria for the diagnosis of amyotrophic lateral sclerosis. Amyotroph Lateral Scler Other Motor Neuron Disord. 2000;1:293-9.

10. Saberi S, Stauffer JE, Schulte DJ, Ravits J. Neuropathology of amyotrophic lateral sclerosis and its variants. Neurol Clin. 2015;33:855-76.

11. Sugiyama A, Sato N, Kimura Y, Maekawa T, Enokizono M, Saito Y, Takahashi $\mathrm{Y}$, Matsuda H, Kuwabara S. MR imaging features of the cerebellum in adult-onset neuronal intranuclear inclusion disease: 8 cases. Am J Neuroradiol. 2017:38:2100-4.

12. Okamura S, Takahashi M, Abe K, Inaba A, Sone J, Orimo J. A case of neuronal intranuclear inclusion disease with recurrent vomiting and without apparent DWI abnormality for the first seven years. Heliyon. 2020;6:e04675. 
13. Takeda T, Uchihara T, Mochizuki Y, Ishihara A, Nakamura A, Sasaki S, Uchiyama S, Iwata M, Mizutani T. Supranuclear ophthalmoparesis and vacuolar degeneration of the cerebral white matter in amyotrophic lateral sclerosis: a clinicopathological study. Amyotroph Lateral Scler. 2012;13:74-83.

14. Sone J, Hishikawa N, Koike H, Hattori N, Hirayama M, Nagamatsu M, Yamamoto M, Tanaka F, Yoshida M, Hashizume Y, Imamura H, Yamada E, Sobue G. Neuronal intranuclear hyaline inclusion disease showing motorsensory and autonomic neuropathy. Neurology. 2005;65:1538-43.

15. de Carvalho M, Swash M. Nerve conduction studies in amyotrophic lateral sclerosis. Muscle Nerve. 2000;23:344-52.

16. Theys PA, Peeters E, Robberecht W. Evolution of motor and sensory deficits in amyotrophic lateral sclerosis setimated by neurophysiological techniques. J Neurol. 1999:246:438-42.

17. Hammad M, Silva A, Glass J, Sladky JT, Benatar M. Clinical, electrophysiologic, and pathologic evidence for sensory abnormalities in ALS. Neurology. 2007;69:2236-42.

18. Pugdahl K, Fuglsang-Frederiksen A, de Carvalho M, Johnsen B, Fawcett PR, Labarre-Vila A, Liguori R, Nix WA, Schofield IS. Generalised sensory system abnormalities in amyotrophic lateral sclerosis: a European multicentre study. J Neurol Neurosurg Psychiatry. 2007:78:746-9.

19. Gelpi E, Botta-Orfila T, Bodi L, Marti S, Kovacs G, Grau-Rivera O, Lozano M, Sánchez-Valle R, Muñoz E, Valldeoriola F, Pagonabarraga J, Tartaglia GG, Milà M. Neuronal intranuclear (hyaline) inclusion disease and fragile X-associated tremor/ataxia syndrome: a morophological and molecular dilemma. Brain. 2017;140:e51.

20. Padilha IG, Nunes RH, Scortegagna FA, Pedroso JL, Marussi VH, Rodrigues Gonçalves MR, Barsottini OGP, da Rocha AJ. MR imaging features of adultonset neuronal intranuclear inclusion disease may be indistinguishable from fragile $X$-associated tremor/ataxia syndrome. Am J Neuroradiol. 2018;39:E100-1.
21. Sone J, Nakamura T, Koike H, Katsuno M, Tanaka F, Iwasaki Y, Yoshida M, Sobue G. Reply: Neuronal intranuclear (hyaline) inclusion disease and fragile $\mathrm{X}$-associated tremor/ataxia syndrome: a morphological and molecular dilemma. Brain. 2017;140:e52.

22. Greco CM, Berman RF, Martin RM, Tassone F, Schwartz PH, Chang A, Trapp BD, Iwahashi C, Brunberg J, Grigsby J, Hessl D, Becker EJ, Papazian J, Leehey MA, Hagerman RJ, Hagerman PJ. Neuropathology of fragile X-associated tremor/ataxia syndrome (FXTAS). Brain. 2006;129:243-55.

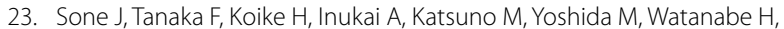
Sobue G. Skin biopsy is useful for the antemortem diagnosis of neuronal intranuclear inclusion disease. Neurology. 2011;76:1372-6.

24. Sone J, Kitagawa N, Sugawara E, Iguchi M, Nakamura R, Koike H, Iwasaki Y, Yoshida M, Takahashi T, Chiba S, Katsuno M, Tanaka F, Sobue G. Neuronal intranuclear inclusion disease cases with leukoencephalopathy diagnosed via skin biopsy. J Neurol Neurosurg Psychiatry. 2014;85:354-6.

\section{Publisher's Note}

Springer Nature remains neutral with regard to jurisdictional claims in published maps and institutional affiliations.
Ready to submit your research? Choose BMC and benefit from:

- fast, convenient online submission

- thorough peer review by experienced researchers in your field

- rapid publication on acceptance

- support for research data, including large and complex data types

- gold Open Access which fosters wider collaboration and increased citations

- maximum visibility for your research: over $100 \mathrm{M}$ website views per year

At BMC, research is always in progress.

Learn more biomedcentral.com/submissions 